\title{
PLATO: PLAnetary Transits and Oscillations of stars
}

\author{
Claude Catala $\cdot$ The PLATO Consortium
}

Received: 2 November 2007 / Accepted: 6 August 2008 / Published online: 16 September 2008 (C) Springer Science + Business Media B.V. 2008

\begin{abstract}
The PLAnetary Transits and Oscillations of stars Mission (PLATO), presented to ESA in the framework of its "Cosmic Vision" programme, will detect and characterize exoplanets by means of their transit signature in front of a very large sample of bright stars, and measure the seismic oscillations of the parent stars orbited by these planets in order to understand the properties of the exoplanetary systems. PLATO is the next-generation planet finder, building on the accomplishments of CoRoT and Kepler: i) it will observe significantly more stars, $i i$ ) its targets will be 2 to 3 magnitudes brighter (hence the precision of the measurements will be correspondingly greater as will be those of post-detection investigations, e.g. spectroscopy, asteroseismology, and eventually imaging), iii) it will be capable of observing significantly smaller exoplanets. The space-based observations will be complemented by groundand space-based follow-up observations. These goals will be achieved by a long-term (4 years), high-precision, high-time-resolution, high-duty-cycle monitoring in visible photometry of a sample of more than 100,000 relatively bright $\left(\mathrm{m}_{V} \leq 12\right)$ stars and another 400,000 down to $\mathrm{m}_{V}=14$. Two different mission concepts are proposed for PLATO: $i$ ) a "staring" concept with 100 small, very wide-field telescopes, assembled on a single platform and all looking at the same $26^{\circ}$ diameter field, and $i$ ) a "spinning" concept with three moderate-size telescopes covering more than 1400 degree $^{2}$.
\end{abstract}

See http://lesia.obspm.fr/cosmicvision/plato/pages/consortium.html for The PLATO Consortium.

C. Catala $(\varangle) \cdot$ The PLATO Consortium

LESIA, Observatoire de Paris, CNRS,

Université Pierre et Marie Curie, Université Paris Diderot,

Place Jules Janssen, 92190 Meudon, France

e-mail: Claude.Catala@obspm.fr

URL: http://www.lesia.obspm.fr/cosmicvision/plato/ 
Keywords Exoplanets • Asteroseismology • Planet evolution •

Stellar evolution - Stellar structure $\cdot$ Photometry

\section{Introduction}

The question of the existence of life beyond Earth has been of concern to humanity for several thousand years. In this context, the search for and study of planetary systems around other stars, and in particular the search for life signatures in exoplanetary systems, is a prerequisite towards generalising our understanding about the distribution of life in the Universe and how it may have arisen on Earth.

Today, one decade after the discovery of the first giant exoplanet, we are entering a new era with the recent launch of CoRoT in December 2006 [2], and with the prospect of the Kepler launch in 2009 [3]. These missions will certainly discover terrestrial exoplanets, and will allow us to start studying the distribution of planet sizes and orbits down to Earth-sized bodies, but they both have their limitations in terms of minimum planet size, maximum orbital period, number of detected exoplanets and capability of further characterization of exoplanet and their host stars. Clearly, we need to overcome these limitations and take the next step in exoplanet detection and characterization.

Most importantly, in addition to the detection of a large number of exoplanets, we need to determine the characteristics of their host stars, such as radius, mass, age, and element abundances. The radii and masses of the host stars must be measured accurately in order to provide a precise measurement of sizes and masses of the detected planets, while the ages of the central stars will provide us with an estimate of the ages of their planetary systems. One of the most powerful tools for this characterization is asteroseismology.

Asteroseismology of a large sample of stars all across the HR diagram can also bring us a full and deep understanding of stellar evolution, which is central to astrophysics. In this area, a first important step will be taken by CoRoT, while Kepler will also include an asteroseismology programme, but they will both provide seismic data for only a modest number of targets, and a next generation mission is clearly needed.

The basic goals of the PLATO mission are therefore:

- to open a new way in exoplanetary science, by providing a full statistical analysis of exoplanetary systems around stars that are bright and nearby enough to allow for simultaneous and/or later detailed studies of their host stars;

- to extend by a factor $\approx 4$ the statistical analysis of exoplanetary systems initiated with Kepler, by surveying many more stars down to a magnitude allowing for the detection of Earth-sized planets; 
- to perform seismic analysis for a very large sample of stars all across the HR diagram, including those with detected exoplanetary systems.

Understanding the processes of star and planet evolution, by the study of stellar interiors and the distribution of planetary systems will constitute a major step for future progress in most areas of astrophysics and in the scientific and philosophical approaches towards the origin of life in the Universe. It is the cornerstone that will bring us from the first, still fairly unsystematic planet discoveries towards a systematic survey, providing a global understanding of the richness and diversity of the "Other Worlds" that populate the Universe.

This papers presents a summarized description of the PLATO mission, as it was submitted to the ESA Cosmic Vision programme in June 2007. Note that the instrumental concept has significantly evolved since then (see http://lesia.obspm.fr/cosmicvision/plato). Section 2 describes the main science objectives of the mission, first in the area of exoplanet science (Section 2.2), then in that of stellar internal structure and evolution (Section 2.3). The tools to be used by PLATO in these two areas (photometric transits and asteroseismology) are discussed in Sections 2.4 and 2.5. The observational requirements are then discussed in Section 3. The PLATO concept also involves an important ground- and space-based follow-up component, which is described in Section 4. Section 5 presents the two instrumental concepts proposed for this mission ("staring" concept in Section 5.1, "spinning" concept in Section 5.2). A comparison with existing or planned missions in the same area is given in Section 6.

\section{Science objectives: evolution of stars and their planetary systems}

\subsection{Studying stars and their planets}

In our conception of planetary systems, the formation and evolution of both components, stars and planets, are intricately related. Stars and planets are born together from the same parental material, and therefore share a common initial history. In particular the initial protoplanetary disc and its central stellar core have the same chemical composition and their respective angular momentum reflects the angular momentum distribution in the protostellar/protoplanetary nebula. Also, in the early phases of evolution, young stars exchange angular momentum with their accretion protoplanetary discs, which eventually evolve into planets and transfer their angular momentum to planet orbits.

Even later in the evolution, various processes occur that result in mutual interactions between the stars and their planets. The stellar radiation flux obviously impacts the planet atmospheres, while particle bombardment of the planet by the stellar winds can also affect the chemical and biological evolution of the planets. Planets can also influence their parent stars, e.g. by colliding with 
them, and enriching them in various chemical elements [12]. Giant planets in close-in orbits can also influence their star's rotation via tidal effects.

The study of planetary system evolution thus must be considered as a whole and it cannot be separated from that of stellar evolution. We cannot understand how planets are formed and how they evolve without a proper knowledge of stellar formation and evolution. We cannot characterize planetary systems without characterizing precisely their host star. The basic philosophy behind the PLATO mission is precisely to study complete planetary systems composed of planets and their host stars, these two components being observed together with the same technique.

\subsection{Evolution of planetary systems}

Our understanding of planetary system formation and evolution is insufficient. Detections of giant exoplanets have revealed a large variety and complexity of configurations in exoplanetary systems, which was totally unexpected. Major questions and uncertainties remain, which hamper our progress in this area.

The true distribution of characteristics of exoplanets and of their orbits is unknown, with current knowledge strongly biased from the detectable subsample. In particular, we do not know the distribution of planets with sizes and masses significantly smaller than those of gaseous giant planets. The extension of our investigation of exoplanets toward lower masses, down to terrestrial planets, may reveal further surprises.

The basic goal of PLATO is to provide a large sample of exoplanets around bright stars, spanning a wide range of orbits, sizes and masses, and to measure precisely and reliably their orbital parameters, sizes, masses and ages. This requires a detailed characterization of their central stars, involving both seismic observations with PLATO and ground-based support observations, allowing us to measure all their fundamental parameters, including mass, radius, age, temperature, chemical composition, rotation. Exoplanetary transit techniques indeed give access to the ratio of planet to star radii, so that the planet sizes cannot be determined if the star radii are not perfectly known. Similarly, radial velocity techniques, even when the inclination angle is known, provide the ratio of planet to star masses, and a good measurement of the star's mass is needed. Star radii and masses are usually estimated by locating the star in the HR diagram, which is imprecise and often unreliable. Finally, the understanding of exoplanetary system evolution requires an estimate of their ages, which can only be obtained by a measurement of the age of their central stars.

Such an approach is beyond our capabilities for most of the planets that will be discovered by CoRoT and Kepler, which are orbiting stars that are too distant and too faint for such a detailed characterization, but is within reach of PLATO, which focuses on stars that are bright and nearby.

Moreover, a full statistical description of exoplanetary systems, down to masses and sizes of a fraction of those of the Earth, is a prerequisite for 
any decisive advance in the field of planetary formation and evolution. It is therefore necessary to extend significantly the sample of detected exoplanets beyond CoRoT and Kepler, which is also an objective of PLATO.

\subsection{Stellar evolution}

Theory of stellar evolution has undergone major progress in the last decades. However, in spite of the progress in our understanding of microscopic physics in stellar interiors, our description of some physical processes controlling stellar structure and evolution is subject to major uncertainties. Convection and various other mixing and transport processes are poorly understood and yet play a major role in stellar evolution, determining evolution timescales, and must be taken into account for measuring stellar ages. Our current poor knowledge of most of these processes is usually compensated in our modeling by some poorly constrained parameterisation, and therefore the resulting stellar ages are strongly model dependent and unreliable.

One of the consequences of this unsatisfactory modeling is that the ages of the oldest globular clusters are still very uncertain, and for some values of the model free parameters can still be higher than the estimated age of the Universe $[8,14,23]$. Additionally, the relatively large adopted value of the core overshooting parameter needed to fit young open cluster data [15] is in contradiction with recent asteroseismic estimates for this parameter for field $\beta$ Cephei stars $[1,16]$. This clearly points out that our current knowledge of convective and rotational mixing processes inside massive stars is very incomplete, resulting in huge uncertainties in stellar masses and ages of supernova progenitors. Uncertainties in convective overshooting can lead to uncertainties in the ages of open clusters up to a factor of two [17]. Considering these difficulties, it is clear that the age ladder of the Universe, which rests on stellar age estimates, is still highly unreliable.

Our modeling of stellar interiors and stellar evolution therefore needs to be seriously improved. The situation for the Sun has evolved considerably with the advent of helioseismology, which has provided precise insight into the properties of the solar interior [7]. Based on this very positive experience, it is clear that asteroseismic investigations, i.e. measurements of oscillation frequencies, amplitudes and lifetimes of a large number of stars of various masses and ages constitute the only and necessary tool to constrain efficiently our modeling of stellar interiors, and improve our understanding of stellar evolution [20].

The pioneering CoRoT space mission is bringing us essential information to progress in this area, by providing high precision asteroseismic measurements for a few dozen stars distributed in several regions of the HR diagram. The Kepler mission will also include an asteroseismology programme. However, these first measurements will not concern essential classes of stars, such as members of open clusters, old population II stars, which would constitute major targets for such investigations. A better and more complete exploration 
of seismic properties of various classes of stars, sampling all stellar parameters (mass, age, rotation, chemical composition) is necessary. Such is the goal of PLATO.

\subsection{Detection and characterization of planetary transits}

Planetary transits can be detected through high precision photometric monitoring, as illustrated by Fig. 1. A planetary transit in front of a stellar disc causes a decrease of the photometric signal $d=\left(R_{p} / R_{*}\right)^{2}$, where $R_{p}$ and $R_{*}$ are the radius of the planet and of the star, respectively. For a star like the Sun, the typical relative variations are $10^{-4}$, with 13 hour duration for $1 \mathrm{AU}$ orbits, for Earth-size planets, and $10^{-2}$ for Jupiter-size bodies. Observations of recurring planetary transits can be used to measure the orbital period $P$, and therefore the semi-major axis of the orbit, by applying Kepler's third law.

The photometric method has the unique ability to determine the ratio of planet to star radius from the transit's depth. It also has the potential to detect small, Earth-sized planets, and is not limited to slowly rotating stars, like the radial velocity technique.

Figure 2 clearly shows that the search for terrestrial-size planets (especially those in the habitable zone) is the unique domain of space-based photometry: (space-based) astrometry can tackle higher-mass, longer-period planets, while the radial velocity searches excel for the shorter-period giant planets.

The main objective of PLATO for exoplanetary science being to detect planets of all sizes, around all types of stars, and to characterize them completely, transit observations therefore constitute the best and the most unbiased technique.

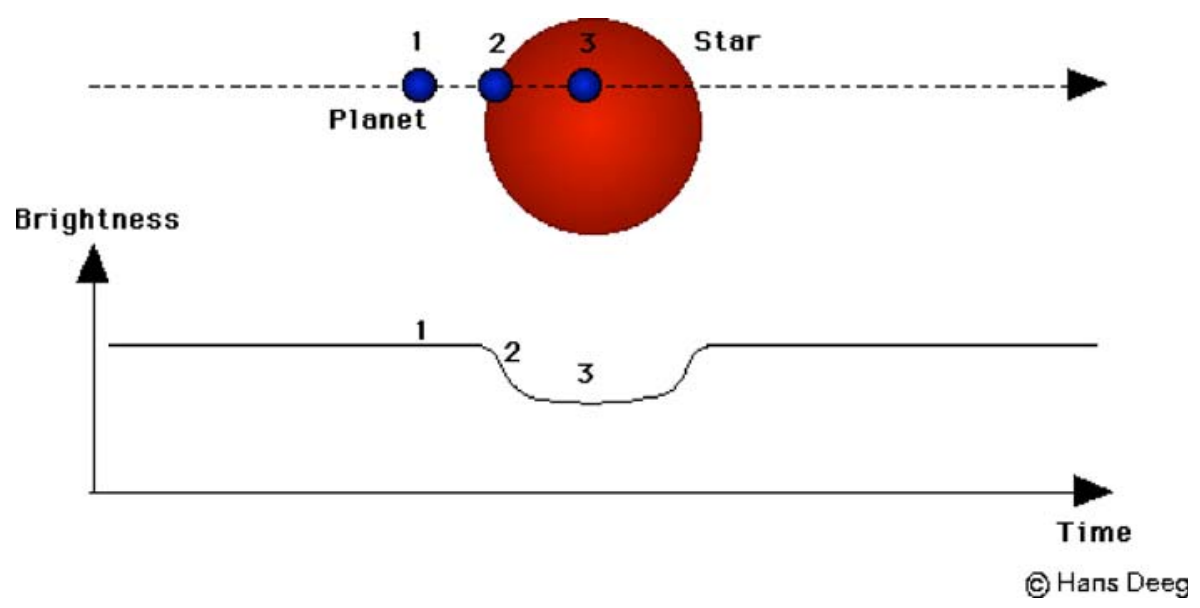

Fig. 1 Schematic illustration of the dynamics of a planetary transit and the corresponding light curve 
Fig. 2 The capabilities of planetary search methods, shown in the orbital period versus planetary mass domain. dots: solar-system planets; triangles: extra-solar planets discovered up to now; dotted lines: astrometric searches; dashed lines: radial velocity searches; horizontal dashed-dotted lines: photometric transit searches. As can be seen, individual transits from Earths can be detected with accurate $\left(10^{-4}\right)$ photometric searches (the type of data PLATO will naturally produce), while they are inaccessible for either of the other methods

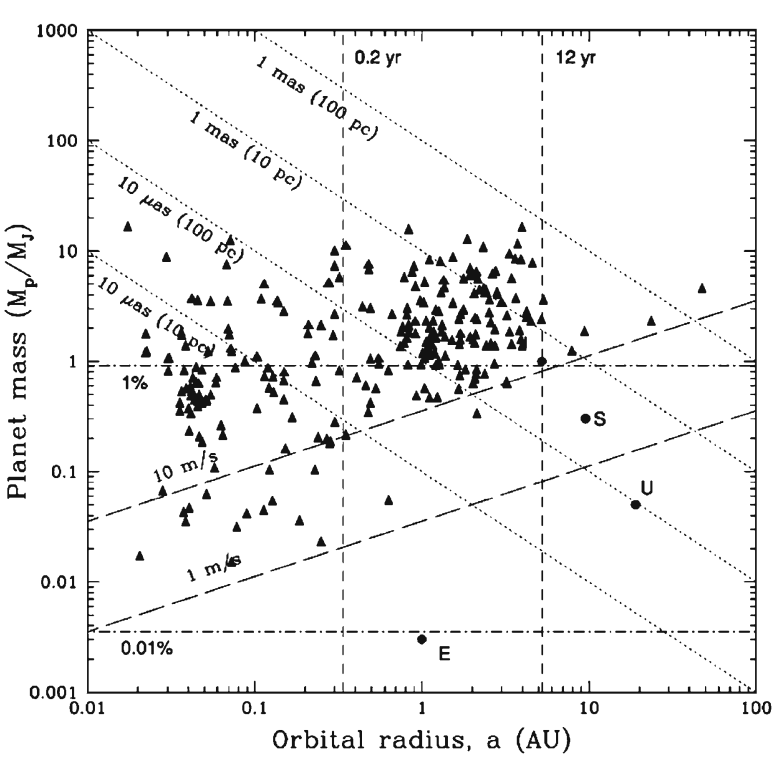

\subsection{Asteroseismology}

Oscillating stars can be found almost everywhere in the HR diagram (Fig. 3). Measurement of a set of oscillation frequencies enables us to infer their internal structure and hence state of evolution. Each frequency senses a different weighted function of the stellar interior. A set of frequencies can yield detailed knowledge of the internal structure, enabling us to deduce the state of evolution and hence the age.

The power of such analyses has been forcefully demonstrated in the solar case, where helioseismology has provided dramatic insight into the properties of the solar interior. Through inversion of extensive sets of frequencies it has been possible to determine the sound speed in most of the Sun, hence testing in detail models of solar structure. This has revealed anomalies below the outer convective zone and just outside the solar core, and also allowed investigation of subtle features of the thermodynamic properties of matter in the Sun, as well as a precise determination of the solar envelope helium abundance. The general agreement between the inferred structure of the solar core and normal solar models strongly indicates that the solution to the solar neutrino problem must result from previously unsuspected properties of the neutrinos, rather than deficiencies in the solar models. Analyses of frequency splittings have yielded detailed determinations of the internal angular velocity. Many of these results are based on the excellent observations obtained from the ESA/NASA $\mathrm{SOHO}$ mission.

In contrast to helioseismology, seismology of distant stars is restricted to low-degree modes, but it is just these modes that penetrate into the deep interior and therefore convey information on the interior structure and state 
Fig. 3 Schematic location of various classes of pulsating stars in the HertzsprungRussell diagram. The solid lines show evolutionary tracks for stars of masses (from the top) of 20, 12, 7, 4, 3, 2 and 1 solar masses

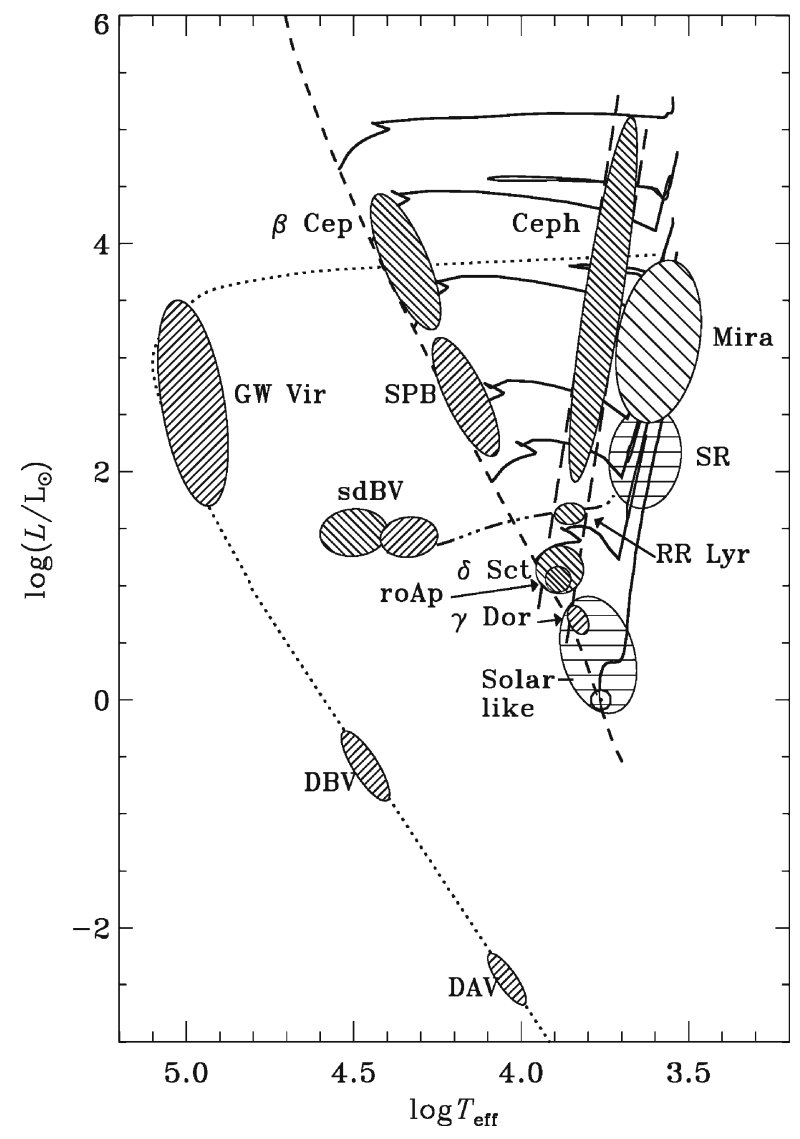

of evolution. Furthermore, the comparatively limited set of modes accessible in stars is compensated by the richness of stellar properties that may be investigated, far beyond the properties of the solar interior, such as the mass and radii of convective cores, rapid rotation and the variation with mass and chemical composition. This will, for example, allow detailed investigations of the uncertain mixing processes associated with convective cores in mainsequence stars, of great importance to their subsequent evolution.

Members of open clusters present a specific interest. Their uniform chemical composition and age, and nearly common distance, provide very stringent constraints on the modeling, increasing the information obtained from the oscillation frequencies. In young clusters, we may in particular observe the $\beta$ Cephei stars, and bring important constraints on the evolution of massive stars. In the older clusters, oscillations in sub-giants (similar to those observed in $\eta$ Bootis [5]) can be studied. Therefore, the asteroseismic analysis of members of open clusters chosen to sample an age sequence, will allow us to constrain severely stellar evolution modeling. 
Also, the properties of the metal-poor stars (Pop. II stars) are of great interest; these are expected to be amongst the oldest in the Galaxy, often similar to the stars that are used for age determinations of globular clusters, and may provide important limits on the age of the Galaxy and, by implication, the Universe.

\section{Required observations}

\subsection{Stellar sample}

The PLATO science objectives require long uninterrupted high precision photometric monitoring of a large sample of stars. The primary stellar sample for PLATO will be a set of at least 100,000 stars brighter than $\mathrm{m}_{V}=11-12$, all observed simultaneously and continuously for several years, at ultra-high precision. In addition to this main sample, for which both transit search and asteroseismology will be performed, an extended sample of 400,000 fainter stars, down to $\mathrm{m}_{V}=14$, will be observed to a sufficient precision to detect the transits of Earth-sized planets.

This requires to survey of a very wide field, typically wider than about 300 square degrees.

Moreover, as mentioned earlier, asteroseismic observations of stars that are members of open clusters, chosen to offer a complete sequence of ages, as well as old population II stars, will be of major interest, and such targets will be included in the programme.

\subsection{Photometric noise level}

In order to detect transits of earth-size planets in front of cool stars, at more than $4 \sigma$, we need to obtain a photometric noise level lower than about $2.5 \times 10^{-5}$ in $12 \mathrm{~h}$, i.e. about $8 \times 10^{-5}$ in $1 \mathrm{~h}$. However, this requirement must be considered as the minimum one, since the detection of planets with sizes smaller than the Earth's, the detection of Earth-sized planets in front of stars hotter than the Sun, and the measurement of several points across the transits would be of major interest. We will set as a goal specification a photometric noise level of $2.5 \times 10^{-5}$ in $1 \mathrm{~h}$, allowing us for instance to measure about ten points across the transits, and therefore to characterize them with high reliability and precision. Such a low noise level would also allow us to detect Earth-sized planets in front of $2 \mathrm{R}_{\odot}$ stars, which are in principle less active and therefore less intrinsically variable than solar twins, so that the detection of such exoplanets may in fact be easier than for solar-Earth twin systems; we would therefore extend to hotter stars the statistics of terrestrial exoplanets.

Figure 4 shows simulated light curves with various levels of noise, all including a $12 \mathrm{~h}$ transit with a depth of $10^{-4}$, illustrating the absolute need for a noise level better than $8 \times 10^{-5}$ in one hour for detecting such a transit and 


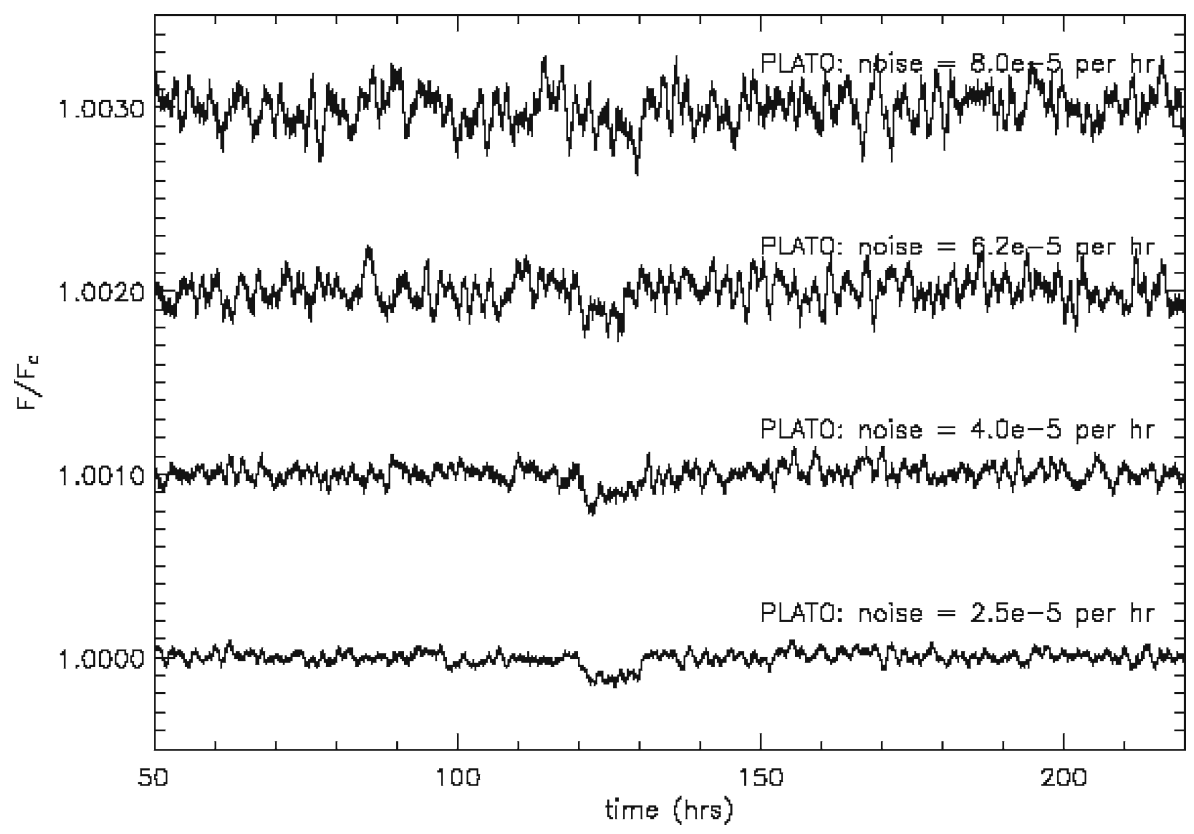

Fig. 4 Simulated light curves assuming various noise levels, and including one single transit with a depth of $10^{-4}$ and a duration of $12 \mathrm{~h}$. The signal from three consecutive transits has been averaged. The time sampling is $32 \mathrm{~s}$, but the light curves have been smoothed to $1 \mathrm{~h}$

the potential for precise transit characterization at a noise level of $2.5 \times 10^{-5}$ in $1 \mathrm{~h}$.

Seismic investigations of planet host stars require that we should be able to detect individual $p$-mode oscillations in cool dwarf stars as faint as $\mathrm{m}_{V}=11$. This means that the total photometric noise in the observations must remain below $\simeq 1 \mathrm{ppm}$ after approximately 30 days of observation, down to $\mathrm{m}_{V}=$ $11-12$, in the whole frequency range of interest, i.e. from 0.1 to $10 \mathrm{mHz}$. Figure 5 shows a portion of the simulated power spectrum for a $1.2 M_{\odot}$ star observed for 50 days with such a photometric noise, as well as that expected for brighter stars leading to lower noise levels. The spectrum was generated with a simulator built in preparation for CoRoT, including photon noise and stellar granulation noise, and its results have been successfully compared to the early results of CoRoT.

The noise levels requirements of $2.5 \times 10^{-5}$ in $1 \mathrm{~h}$ and one ppm in 30 days are very similar. These photometric noise requirements impose that $i$ ) the photon flux of the target stars is sufficiently high to ensure that photon noise complies with the final noise specifications, implying the use of a large collecting area, and $i i$ ) that all other sources of noise remain well below photon noise in the frequency range of interest.

The above noise requirements, applied for stars with $\mathrm{m}_{V}=11-12$, imply a photo-electron flux of the order $4 \times 10^{5} \mathrm{~s}^{-1}$ at this magnitude, which requires 


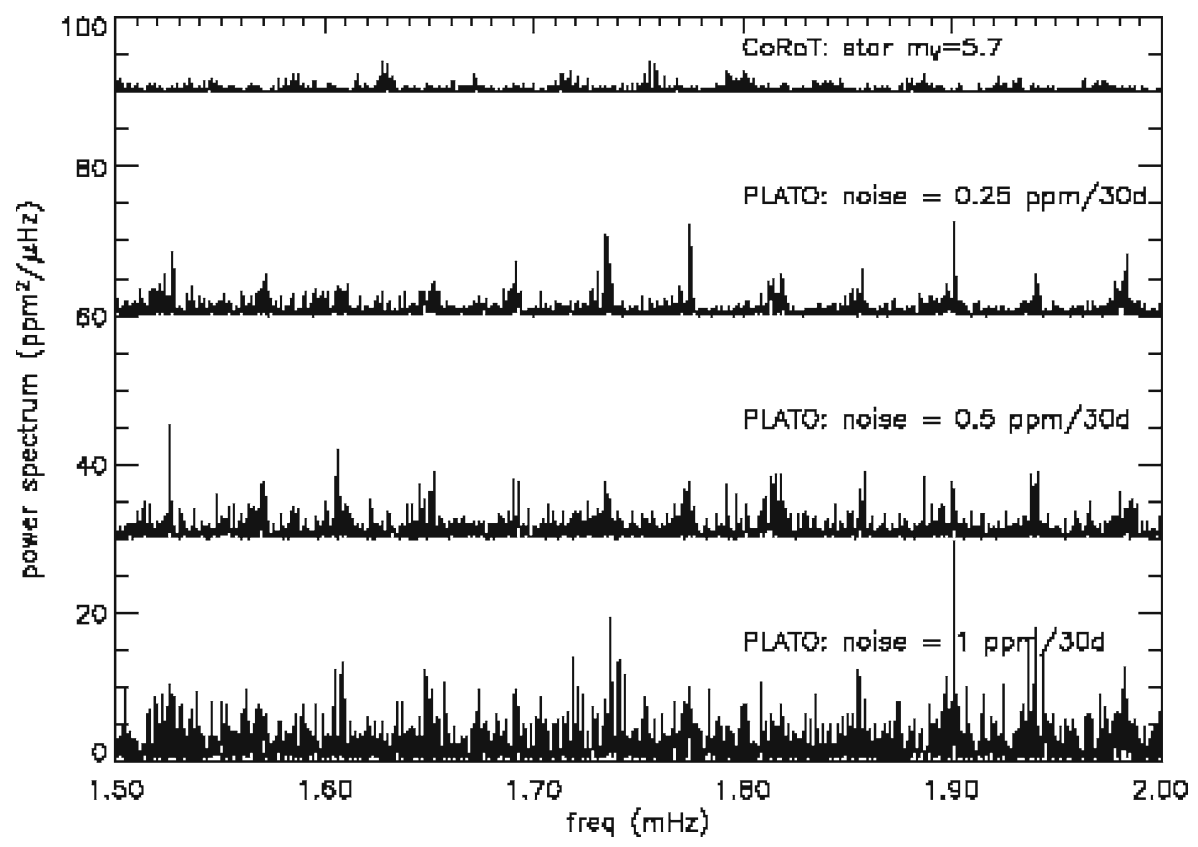

Fig. 5 Expected power spectrum for a $1.2 M_{\odot}$ star, observed for 50 days, with a noise level of one ppm in 30 days (lower curve). Such a noise level will typically be obtained at $\mathrm{m}_{V}=11.5$ or 12 with PLATO, depending on the details of the instrumental design. The next two curves correspond to better noise levels of 0.5 and $0.25 \mathrm{ppm}$ in 30 days, achievable with PLATO at $\mathrm{m}_{V}=10$ and 8.5 , respectively. The upper curve shows a preliminary result obtained with CoRoT in 50 days on a solar-type star with $\mathrm{m}_{V}=5.7$, which translates to $\mathrm{m}_{V}=8.5$ for PLATO when the ratio of collecting areas is taken into account. This CoRoT result demonstrates that the noise levels introduced in the simulation are realistic and that solar-like oscillations can indeed be easily observed in photometry from space

the use of an optical design with a high overall throughput and a large effective collective area.

The overall optics + detector throughput can reasonably be expected of the order of 0.6 in average over a bandpass of $500 \mathrm{~nm}$, using high quantum efficiency detectors, such as, e.g., thinned backside illuminated CCDs, as well as efficient, optimally coated optics. Under these assumptions, it can be calculated that an effective total collecting area of $0.8 \mathrm{~m}^{2}$ is necessary to yield the required photo-electron flux down to $\mathrm{m}_{V}=12$, while a collecting area of $0.7 \mathrm{~m}^{2}$ would comply with the flux requirement at $\mathrm{m}_{V}=11.5$.

\subsection{Non photonic sources of noise}

We must ensure that additional sources of noise remain significantly below photon noise. Until a full analysis of all environmental and instrumental perturbations is carried out, we will specify that any given source of noise 
must remain at least three times below photon noise for stars with $\mathrm{m}_{V}=11$. We provide below some hints concerning the expected two major sources of perturbations, satellite jitter and thermal instability, but a thorough study of all possible other sources of noise must be carried out when designing the instrument and the mission during assessment, phase-A and B1.

Satellite jitter introduces photometric noise, as drifts in the star's position will change the illumination pattern of the detector, whose pixels do not have exactly the same sensitivity. While a large fraction of the induced noise can be calibrated out, as demonstrated by CoRoT results, a residual noise will still be present, and impose pointing stability requirements. At $\mathrm{m}_{V}=11$, the photon noise will be of the order of $2 \times 10^{-4}$ per minute, and jitter noise must remain about three times below this value, i.e. below $6 \times 10^{-5}$ per minute. A detailed study, depending on the instrument design, is necessary to figure out how this requirement translates into pointing stability specifications, but order of magnitude estimates, as well as experience gained with previous missions such as CoRoT, indicate that a pointing stability of about $0.2 \operatorname{arcsec}$ on timescales of minutes will be needed.

Variation in temperature of both the telescopes and the CCD detectors will lead, directly or indirectly, to changes in the apparent signal level from the target stars. The top level requirement for these perturbations is that the equivalent noise level induced by thermal changes must be three times below the photon noise level at $\mathrm{m}_{V}=11$. While detailed a posteriori corrections can drastically improve the resulting noise, as demonstrated by CoRoT preliminary results, a full study taking into account the details of the instrument design and environmental perturbations must be carried out in order to specify clearly the thermal stability requirements. Again, as a first estimate, we can take advantage of the CoRoT experience, and require that the chip temperature must be stable to $0.1 \mathrm{~K} / \mathrm{h}$, while a thermal stability of a few tenths of a $\mathrm{K}$ per day will be required for the telescope structure.

\subsection{Duration of observations and duty cycle}

The duration of the observations needs to be longer than three years, so that at least three consecutive transits for Sun-Earth analogs can be detected during the mission lifetime. A goal specification for the duration should be four years, allowing us to detect planets in more distant orbits than the Earth's and also increasing the transit detection probability.

A monitoring time of at least a few months (minimum 1 month) is required for the seismic analysis of the programme stars, yielding a precision of about $0.3 \mu \mathrm{Hz}$ for oscillation frequencies of solar-type stars.

The duty cycle of the monitoring needs to be better than $90 \%$, in order to make sure that no transit is missed due to gaps in the data, and also to avoid side lobes in the oscillation spectra. 


\subsection{Colour information}

Intrinsic stellar variability due to activity or other stellar phenomena can lead to variations of the light curves that are similar to those produced by the transits of terrestrial exoplanets. Planet occultations can be discriminated from these intrinsic variations if several successive transits can be observed. In practice, planetary transits are definitely discriminated from intrinsic stellar variations only after three transits, so that the longest orbital period detectable is in average between one third and one half of the total monitoring time.

Light curves in several colours can improve significantly the situation. Planetary transits indeed are achromatic, while all intrinsic variability pattern show some degree of chromatism. Comparing the depths and shapes of the candidate transits in several colours will allow us to distinguish transits from stellar intrinsic variations. Similarly, colour information will allow to discriminate against false positives arising from of stellar configurations involving eclipsing binaries [4], whose vast majority generates a chromatic signal. The level of confidence for candidate transits will therefore be greatly enhanced at first occurrence, hence increasing significantly the range of reachable orbital periods.

In addition to the measurement of oscillation frequencies, asteroseismology requires the identification $(\ell, m)$ of the detected modes. Knowing the $\ell$ identification for the dominant modes of each of the 100,000 bright target stars of PLATO implies a significant reduction of the free parameter space of stellar models and is a requirement to guarantee successful seismic inference of their interior structure parameters and ages.

For oscillations in the asymptotic frequency regime, the derivation of frequency spacings suffices to identify the modes, as in the solar case. For most main-sequence stars excited by the $\kappa$-mechanism, when the modes do not follow particular frequency patterns, the identification of $\ell$ can be achieved by exploiting the difference in amplitude and phase of the mode at different wavelengths [10].

However, whatever the interest of coloured information, we must not compromise photon noise, which is the most important requirement of this mission. The option to measure light curves in several colours must be brought at negligible cost in terms of photon loss. This is why the instrumental concepts presented later envisage to use dispersive elements rather than filters to produce light curves in three colours, in a similar way as what is done with CoRoT.

\subsection{Time sampling}

The duration $\Delta t_{\mathrm{tr}}$ of a transit of a planet with semi-major axis $a$ and orbital period $P$ in front of a star with radius $R_{*}$ is given by $\Delta t_{\mathrm{tr}}=P R_{*} /(a \pi)$. For true Earth analogs $\Delta t_{\text {tr }}=13$ hours. More generally, the duration of a transit around a single star may last from about two hours (a "hot giant" planet around a low-mass star) to over one day, for planets on Jupiter-like orbits (five AU 
distance). Planets in the habitable zone, however, will cause transits lasting between five hours (around M stars) and $15 \mathrm{~h}$ (for F stars), for equatorial transits.

Because individual transits have durations $\geq 2 \mathrm{~h}$, a time sampling of about $10-15 \mathrm{~min}$ is in principle sufficient to detect all types of transits, as well as to measure transit duration and period. However, a higher time resolution is needed in order to accurately time ingress and egress of the planet transits for which the $S / N$ in the light curve will be sufficient. The accurate timing will allow the detection of third bodies, which cause offsets in transit times of a few seconds to about a minute, and will allow to solve ambiguities among possible transit configurations through the determination of ingress and egress time of the planet. In practice, a time sampling of about $30 \mathrm{~s}$ will be necessary to analyse in such detail the detected transits.

The needed time sampling for the asteroseismology objectives can be derived directly from the frequency interval we need to explore, which is from 0.1 to $10 \mathrm{mHz}$. In order to reach $10 \mathrm{mHz}$, the time sampling must be at least twice this frequency, i.e. of the order of $50 \mathrm{~s}$. However, the necessary sampling will strongly depend on the type of stars considered. A sampling of about ten minutes will be sufficient for most stars in the upper HR diagram, such as $\gamma$ Dor, $\delta$ Sct, $\beta$ Cep, SPB (see Fig. 3), while a sampling better than $50 \mathrm{~s}$ will be required for solar-type stars.

In summary, a $30 \mathrm{~s}$ sampling will be needed for the cool stars in the bright sample of 100,000 stars for precise transit analysis and asteroseismology, while a sampling of ten minutes will be sufficient to search for transits in the fainter sample of 400,000 stars, and for asteroseismology of hotter stars. For the small fraction of these faint stars for which a transit will have been detected, a $30 \mathrm{~s}$ sampling will be applied.

\subsection{Dynamical range}

The planet search objectives of PLATO are based on the observation of stars as faint as $\mathrm{m}_{V}=13-14$, but stars as bright as $\mathrm{m}_{V}=6-7$ also need to be monitored for the detection of stellar reflected light on planet atmospheres and also for astrometric detection of planets.

Stars between $\mathrm{m}_{V}=6$ and $\mathrm{m}_{V}=11-12$ also need to be observed at a sufficient level for seismic analysis, i.e. with a noise level below $2.5 \times 10^{-5}$ in one hour.

\section{Follow-up observations}

A vast ground-based programme, in particular to identify real planets from transit candidates, will be an integral part of the overall mission, and will be organized should the mission be selected. Indeed radial velocity observations, as well as ground-based imaging and photometry observations are required in complement to the space photometric data. The ultra-precise photometric 
observations provided by PLATO will also need to be complemented by ground- and space-based follow-up observations, in order to complete the characterization of the detected planetary systems, their central stars, as well as all the stars in the PLATO programme. One of the main advantages of the approach proposed for PLATO will be to focus on bright stars, so that this follow-up will be made easy and efficient.

We give below some relevant examples of follow-up observations that will be needed.

Radial velocity measurements a radial velocity monitoring of the stars with planetary transit candidates detected by PLATO will be necessary in order i) to confirm when needed that the candidate transit was indeed produced by a planet, and ii) to provide a measurement of the planet mass, taking advantage of the accurate knowledge of the star's mass derived from seismic analysis with PLATO. Today's state-of-the-art radial velocity monitoring, such as performed with the HARPS spectrograph at the ESO $3.6 \mathrm{~m}$, can reach precisions of the order of $1 \mathrm{~m} \mathrm{~s}^{-1}$, sufficient to detect the signature of planets with a few Earth masses around solar-type stars [22]. We cannot reasonably hope to lower significantly this detection limit. However, although PLATO will detect many smaller and less massive planets, the planet mass measurements down to a few Earth masses will be crucial for the characterization of a large fraction of the planet sample detected by PLATO. Note that very high resolution and very stable spectrographs, of the same type as HARPS at the ESO $3.6 \mathrm{~m}$, will certainly be available at the time the first Cosmic Vision M mission is flown, and later. For instance, one of the main instruments foreseen for the e-ELT, CODEX, is precisely of this type.

Differential photometry and spectroscopy planets around bright $\left(\mathrm{m}_{V}=4-10\right)$ stars detected with PLATO will have a long-lasting legacy for future follow-up observations to characterize further the nature of extrasolar planets. Photometric and spectroscopic observations of the upper atmosphere levels can be obtained during primary and secondary eclipse of the planets orbiting nearby and bright stars, which are observable at very high signal-to-noise ratio. Several transiting planets in the recent past have already shown the huge potential of such characterizing observations and their impact on our understanding of the formation and evolution of planets. Thermal emission of HD189733b, HD149026b, HD209458b and TrES-1 [9, 11, 13, 18, 24] has been detected with the Spitzer infrared telescope. Infrared spectra of HD189733b $\left(\mathrm{m}_{V}=7.7\right.$ [11] obtained with Spitzer provided upper limits on water and methane absorption. TrES-1 $\left(\mathrm{m}_{V}=11.8\right)$ observations gave upper limits on $\mathrm{CO}$ and water. The best characterized planet so far is HD209458b $\left(\mathrm{m}_{V}=7.7\right)$. In this planet water and silicate clouds [18] have been detected in the infrared with Spitzer, sodium absorption [6] in the optical and HI, OI, and CII [24] in the UV by HST. Future detections of planets around bright stars with PLATO are expected to provide "gold-mine" of targets for further planet characterization. At the time the first M-class Cosmic Vision mission will be flown, several very efficient space- and 
ground-based facilities will be available for these purposes, including JWST for infrared photometry and spectroscopy, and the e-ELT for high resolution spectroscopy in the optical and near IR.

High resolution spectroscopy all stars observed by PLATO will be easily observable in high resolution spectroscopy in the optical and near IR, with various instruments on 4- and 8-m class telescopes (e.g. the VLT). Such observations will be used both in preparation and as follow-up of PLATO, for deriving all fundamental parameters of stars, such as their effective temperature, surface gravity, surface chemical composition, rotation velocity, level of magnetic activity, etc. Such information will be essential, in addition to the asteroseismic parameters derived from PLATO photometry, for refining the modeling of these stars.

Gaia among the various complementary observations for PLATO, those provided by Gaia will play a particularly important role. The precise parallaxes derived by Gaia for all stars observed by PLATO will be used to measure their absolute luminosities and place them accurately on the HR diagram. Also, coupled with interferometric measurements of stellar angular diameters for the most nearby of these stars, these precise parallaxes will give us an independent measurement of the physical diameter of these stars, a very powerful additional constraint for their modeling.

Ground-based interferometry angular diameters of stars out to several tens of parsecs are within reach of present and future ground-based interferometers, such as VLTI. In some cases, these interferometric observations may also be used to directly detect and characterize giant exoplanets around nearby stars, in particular those that will have been identified by PLATO.

Space interferometry finally, future interferometric missions, can use PLATO results to identify the best targets for studying terrestrial planets and their atmospheres. The field surveyed by PLATO is wide enough to include a large number of bright and nearby stars, around which we can hope to detect at least a few transiting terrestrial planets, and in any case PLATO will discover a significant number of giant exoplanets around these nearby stars, either by the reflected stellar light on the planet atmosphere, or by the astrometric measurement of the star's reflex motion.

\section{Instrumental concepts}

Two different instrumental concepts have been studied for the PLATO mission, involving different observational strategies. Note that alternative concepts have also been envisaged, and could be investigated in further studies. Both concepts assume that the instrument is placed by a Soyuz-Fregat launcher 
on a large Lissajous orbit around the L2 point, so that the duty cycle of the observations is very high $(\geq 95 \%)$, and that the duration of the mission is 5 years.

\subsection{The "staring" concept}

\subsubsection{Observation strategy}

In the staring concept, a first phase of the mission, with a duration of typically 3 or 4 years, is spent observing continuously the same field. This field is chosen to provide a sufficient number of stars, as well as to contain stars presenting a specific interest for the seismology objectives of the mission, e.g. open clusters.

During this first staring phase, the field is observed continuously with a duty cycle close to $100 \%$. This staring phase will be followed by a "step and stare" phase of typically one additional year, where the payload will be pointed successively to several additional fields, each one monitored continuously for one to several months. The goal of this second phase will be: $i$ ) to extend to other regions of the galaxy the exoplanet statistics for planets on orbits shorter than a couple of months; and $i$ ) to optimize the sample of stars of the seismology programme.

\subsubsection{Payload}

The science objectives of PLATO require both a very wide field of view (FOV) $\left(\geq 300 \mathrm{deg}^{2}\right)$ and a large collecting area $\left(\geq 0.7 \mathrm{~m}^{2}\right)$. The very wide FOV requires a short focal length yielding a large plate factor, which implies the use of a small pupil, since the optics cannot easily be made faster than $\mathrm{f} / 1.5-\mathrm{f} / 1.3$. In order to comply with the collecting area requirement, we propose to use a large number of identical small size optics, each one coupled to its own focal plane, all of them observing the same stellar field. Figure 6 shows the proposed payload on the recurrent Herschel platform, which is foreseen for this mission.

The majority of these small pupil optics will be devoted to the study of faint $\left(\mathrm{m}_{V} \geq 9\right)$ stars in the field, using an elementary exposure time of $30 \mathrm{sec}$, minimizing the losses during camera readout. For the observation of brighter stars that saturate the detectors with this exposure time, the plan is to use a small number of telescopes, with shorter elementary exposure times $(1 \mathrm{~s})$.

Optical design The concept (Fig. 7) consists of 100 identical axi-symmetric compact cameras, each one covering the same annular FOV between $13^{\circ}$ and $6^{\circ}$ radius. The optical design is based on a One Mirror Anastigmat (OMA) already proposed for the Eddington mission. Its advantage is its capability to work with fast aperture and wide FOV. A second advantage is that the $100 \mathrm{~mm}$ pupil is placed on the first lens of the corrector so that the total mass is minimised by decreasing the lens dimension. On the other hand, the mirror diameter $(240 \mathrm{~mm})$ is larger than the pupil size, due to the pupil scan generated by the FOV $\left(26^{\circ}\right)$. With a focal length of $227 \mathrm{~mm}$, the plate scale is $1.1 \mu \mathrm{m}$ per 
Fig. 6 View of the payload integrated on full recurrent Herschel service module

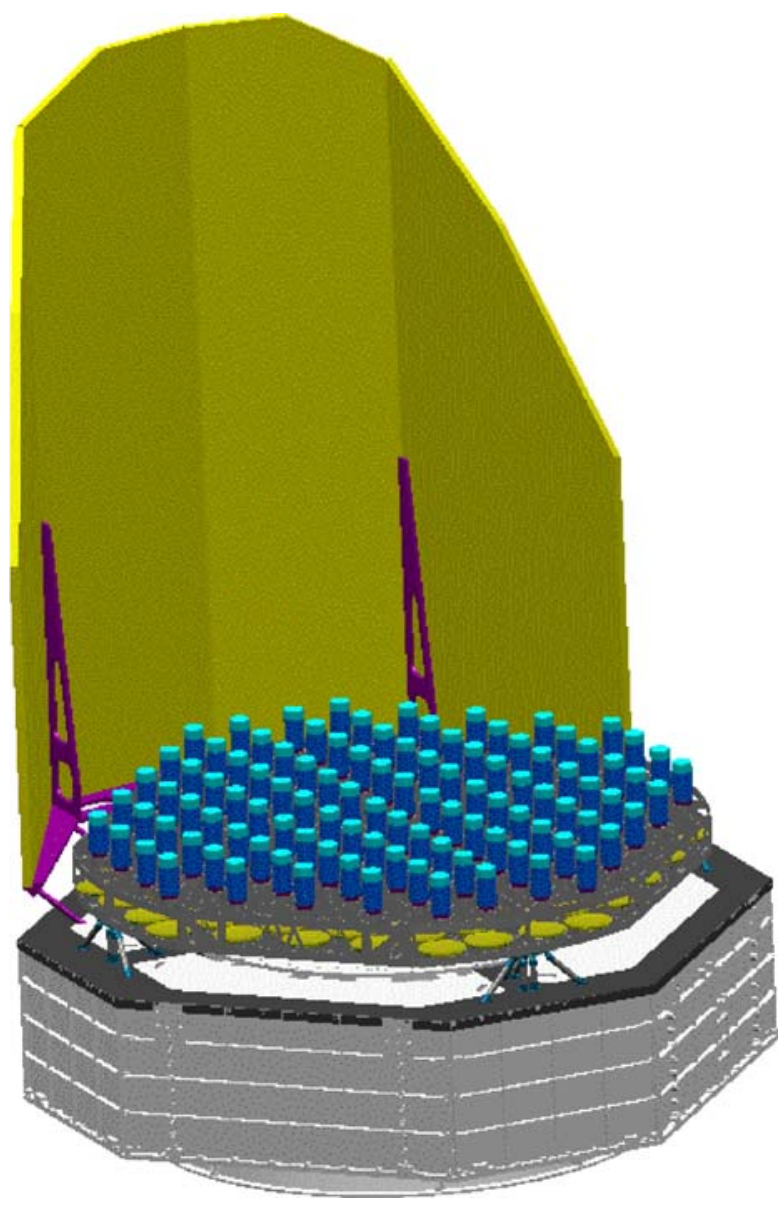

arcsec. The optical performance is optimised to get a homogeneous spot inside this field, when a defocus of $-50 \mu \mathrm{m}$ is applied. The spot size in this case varies between 25 and $35 \mu \mathrm{m}$, i.e. about two to three pixels, across the field.

The colour information can be obtained by introducing a dispersive system in the optical design, for instance a small angle prism. The introduction of a prism in the optical beam, completed by the construction of appropriate numerical masks for the aperture photometry algorithm, will result in the production of light curves in three colours, in a similar way as in CoRoT.

Focal plane concept Each one of the 100 focal planes is made of several identical detectors, with $13 \mu \mathrm{m}$ pixels, corresponding to $10 \operatorname{arcsec}$ on the sky. Many possibilities can be proposed for the actual size of the chips and their accommodation. One of them is given in Fig. 8, based on $800 \times 1800$ pixel CCDs. 
Fig. 7 Optical design of the individual cameras. The prime mirror has a diameter of $240 \mathrm{~mm}$

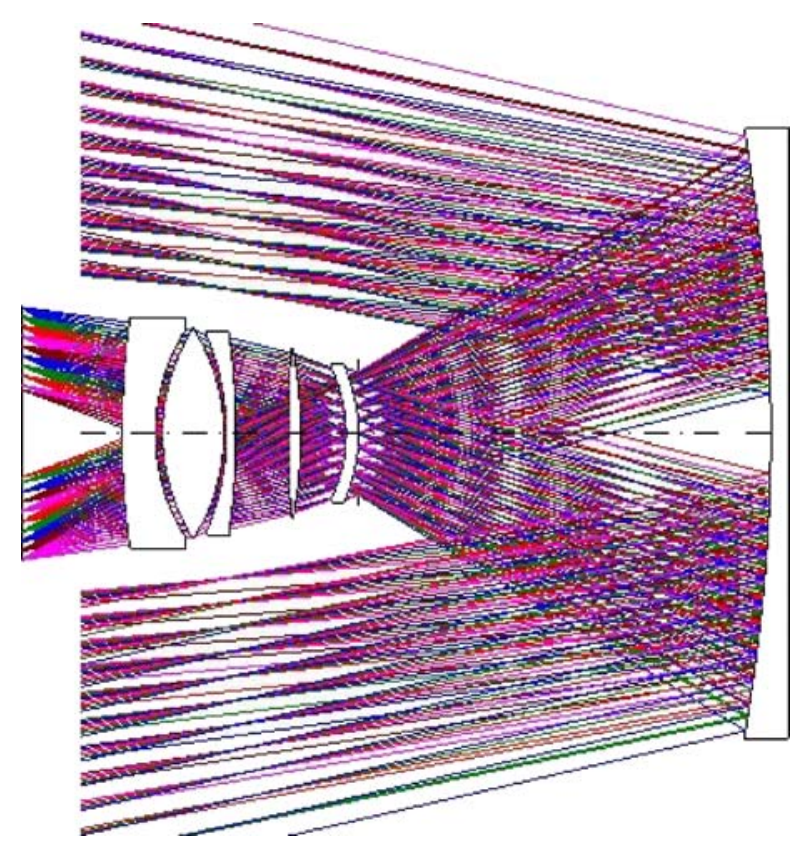

The CCDs are defocused to produce a PSF varying across the field between 2 and 2.5 pixels, i.e. between 20 and 25 arcsec. This defocusing is needed both to avoid saturation while collecting large photon fluxes, and to limit the impact of satellite jitter on the photometric performance.

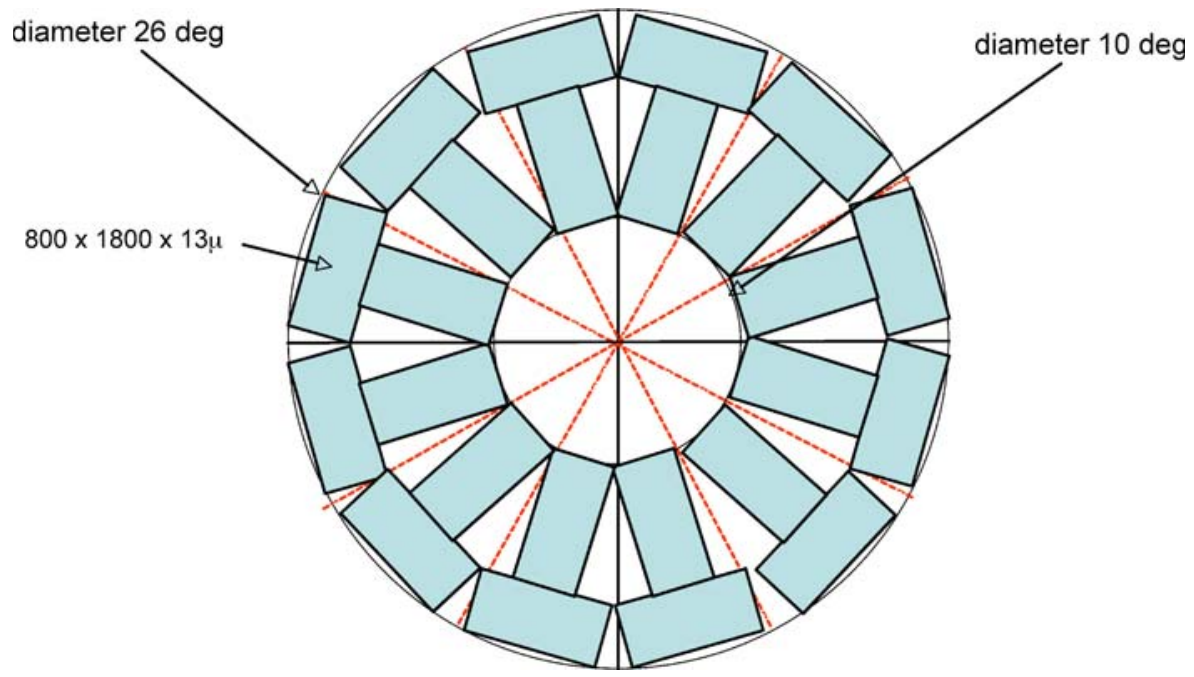

Fig. 8 One possible accommodation for populating the focal plane 
Onboard data processing \& downlink rates Taking into account the inertial pointing of the instrument with stars assigned to keep a constant position on the CCD, the aperture photometry can be performed with fixed photometric masks. To process the huge amount of collected data, it is foreseen to apply the windowing and the individual light-curve and centroid processing as close as possible to each focal plane. With some elementary computing power at immediate proximity of each camera, the amount of data can therefore be reduced from $18 \mathrm{Mbits} / \mathrm{s}$ to roughly $200 \mathrm{kbits} / \mathrm{s}$. This elementary computing function is likely to be performed by local ASIC.

On the ten cameras dedicated to bright stars $\left(\mathrm{m}_{V}\right.$ between 6 and 9$)$, the number of targets will be small and windowing can be applied at CCD level.

Downstream from the complete set of cameras, one or several central processing units (calculators) will multiplex, compare and average the 100 lightcurves for each star, and compress the photometric data. The central processing unit is also in charge of computing the average centroid measurements both for line of sight calibration (jitter correction on light curves) and delivery of payload depointing angles to the SVM AOCS, in a similar way as what has been done on CoRoT or studied for Eddington. Before compression, the mission data rate is estimated at $340 \mathrm{kbits} / \mathrm{s}$. By implementing a DPCM loss-less compression algorithm, expected to be particularly efficient and reliable on photometric time series (small signal variations close to a stable mean value, no scientific correlation between targets), the global data rate between the payload and the SVM is assumed to be close to $140 \mathrm{kbits} / \mathrm{s}$ (compression factor around 2.5). This is fully in line with the current SVM capabilities.

\subsubsection{Expected performance}

The performance of the design presented above can be estimated in terms of number of accessible targets and achievable level of noise.

Because the field obstruction varies significantly across the field, targets with the same magnitude are not observed with the same level of noise across the field. In other words, a given level of noise will corresponds to stars with different magnitudes at different locations in the field. The expected performance can therefore be expressed in terms of number of targets observable with various levels of noise during the initial 3-4 year monitoring. Table 1 indicates these numbers of targets, which have been calculated assuming a template field centred around a position at $\left(210^{\circ},-60^{\circ}\right)$ in ecliptic coordinates. The star numbers were counted using the 2MASS, Tycho-2 and USNO-A2.0 catalogues, with fractions of dwarfs and subgiants derived from the Besançon model [19]. These predictions are compliant with the basic requirements of this mission, since we can observe more than 100,000 stars at a noise level better than $2.6 \times 10^{-5}$ per hour, and up to 360,000 stars with a noise better than about $8 \times 10^{-5}$ per hour.

The proposed design can also achieve a very high photometric and astrometric precision for the observation of very bright stars in the field. Table 2 
Table 1 Number of stars observable with various levels of photometric noise

\begin{tabular}{lllcc}
\hline Noise & & & $\#$ & Mag range \\
\cline { 1 - 2 } $\mathrm{ppm} / \mathrm{hr}$ & $\mathrm{ppm} / 30 \mathrm{~d}$ & Stars & $\begin{array}{c}\text { Dwarfs } \\
\text { +subgiants }\end{array}$ & \\
\hline 10 & 0.4 & 10,000 & 5,200 & $9.3-9.9$ \\
15 & 0.5 & 21,000 & 11,000 & $10.1-10.7$ \\
20 & 0.7 & 49,000 & 25,000 & $10.7-11.3$ \\
26 & 1.0 & 101,000 & 52,000 & $11.3-11.9$ \\
53 & 2.0 & 263,000 & 137,000 & $12.8-13.4$ \\
81 & 3.0 & & 360,000 & $13.7-14.3$ \\
100 & 3.7 & & 410,000 & $14.2-14.7$ \\
\hline
\end{tabular}

summarizes the performance, assuming that stars brighter than $\mathrm{m}_{V}=9$ are monitored with ten telescope units only. The performances summarized in this table show that giant exoplanets on close-in orbits can be detected around bright stars, using the photometric modulation due to scattered stellar light on the planet atmospheres, since the amplitude of the modulation due to stellar light reflected on a giant planet orbiting its star at $0.05 \mathrm{AU}$, with an albedo of 0.4 , is of the order of $10 \mathrm{ppm}$ [21].

Also, the astrometric wobble of the stars produced by exoplanets with Jupiter mass orbiting their stars at typically one AU, placed for instance at $15 \mathrm{pc}$, which is of the order of $60 \mu \mathrm{as}$, will be detectable at least down to $\mathrm{m}_{V}=8$. Note however that this performance estimate is based on photon noise only, and that various potential systematic effects (long term drifts of the line of sight, variability of faint background sources, etc) need to be studied in detail before a solid prediction can be made about the final astrometric precision of PLATO measurements.

\subsubsection{Alignment and test philosophy}

Thanks to the tolerant optical solution in terms of misalignment (small camera) and thanks to the huge number of cameras, a new alignment and verification philosophy can be proposed. The idea is to align each camera (mirror vs lenses) by using a tri-dimensional machine (whose typical accuracy is compliant with the needed tolerances), making the alignment of the 100 cameras easy, fast and cheap.

A new statistical approach for the verification phase can also be proposed. The final performance (encircled energy) can be measured only on a limited number of cameras (e.g. 10 to 20 ) in order to derive the statistical characteristics of the whole instrument. If in the end, up to $10 \%$ of the cameras are out of

Table 2 Bright stars observable with PLATO

\begin{tabular}{|c|c|c|c|c|}
\hline \multirow[t]{2}{*}{$\mathrm{m}_{V}$} & \multirow[t]{2}{*}{ \# Stars } & \multicolumn{2}{|l|}{ Noise } & \multirow{2}{*}{$\begin{array}{l}\text { Astrom. noise/ } \\
30 \mathrm{~d} \text { ( } \mu \text { as) }\end{array}$} \\
\hline & & $\mathrm{ppm} / \mathrm{hr}$ & $\mathrm{ppm} / 30 \mathrm{~d}$ & \\
\hline 6 & 85 & 6.4 & 0.3 & 6 \\
\hline 7 & 270 & 11 & 0.5 & 10 \\
\hline 8 & 810 & 17 & 0.8 & 15 \\
\hline
\end{tabular}


compliance or lost, the impact on the final performance remains very low: the decrease in SNR is only about $5 \%$, which is still acceptable for the mission.

Such statistical approach is also foreseen to be applied for the CCD procurement: if some pixels, rows or columns are not usable, it is not a real problem since statistically, it is quite impossible to have all bad pixels for each camera located on the same sky area. The final manufacturing rejection and the acceptance tests can be largely relaxed inducing a large cost benefit.

\subsection{The "spinning" concept}

\subsubsection{Measurement principle}

The spinning concept is based on the re-use of Gaia Service Module, and on the use of three telescopes of aperture $0.72 \mathrm{~m}^{2}$ and FOV 23 degree $^{2}$ each. The concept enables terrestrial planet detection in solar-type systems for a sample of 144,000 stars brighter than $\mathrm{m}_{V}=11$, by exploring a FOV larger than 1400 degree $^{2}$, and the detection of planets with radii $\leq 4$ earth radii in solar-type systems for more than 2,500,000 stars. Colour measurements can be included for enhancing transit discrimination. The instrument is operated in two modes: the search mode for planet finding and asteroseismology, and the fine observing mode for improving the planet transit measurements and for asteroseismology.

The payload consists in three identical telescopes observing in directions regularly distributed on a great circle. When the entire system is rotated about an axis perpendicular to the great circle with a period $T$, each telescope will scan a band centred on the great circle of width equal to the telescope FOV across scan. Any object located in the scanned band will be regularly observed with a sampling time $T / 3$.

The telescopes have a rectangular collecting aperture $0.9 \times 0.8 \mathrm{~m}^{2}$, and the rotation period is $T=20 \mathrm{~min}$. The use of Three Mirror Anastigmat optics provides a large FOV 5.9 $($ along scan $) \times 3.9^{\circ}($ acros scan $)=23$ degree $^{2}$ per telescope. The design assumes a re-use of Gaia detectors, with a binning of 21 (along scan) $\times 7$ (across scan) pixels, providing a macro-pixel sky resolution of 12.4 arcsec with 3.5-m focal length, and a charge collection capacity of about $3 \times 10^{7}$ e- per macro pixel.

There are two operating modes, the search observing mode and the fine observing mode, which are detailed below, assuming equal time sharing between the two modes.

For the search observing mode, the satellite is rotated so as to scan the galactic disk. This mode explores a FOV of about $1400 \mathrm{deg}^{2}$ for planet detection and enables potential terrestrial planet detection for more than 144,000 stars. It can also achieve asteroseismology of classical pulsators in the upper main sequence, such as e.g. $\delta \mathrm{Scu}, \gamma$ Dor, $\beta \mathrm{Cep}$, Be stars, whose pulsation frequencies are sufficiently low to be well sampled by the spinning procedure. 
For the fine observing mode, the instruments are operated in staring mode for maximizing the observing time on selected areas that have been determined in the search mode. One of the telescopes is pointed to the galactic disk while the two others are exploring new areas of galactic latitudes below $60^{\circ}$. One can observe in fine mode up to one third of the stars that have been measured in search mode (plus extra stars due to the FOV extension and position).

The observation strategy relies on the separation of the year in two parts: one when the spacecraft is rotated to scan the galactic plane permanently and one when the spacecraft has a fixed three-axis control to point one of its telescope towards a selected target field.

The instrument parameters are summarised below.

\begin{tabular}{|l|c|c|}
\hline \multicolumn{3}{|l|}{ Telescopes } \\
\hline Number of telescopes & 3 & \\
\hline Diamètre ALong scan (AL) & 900 & $\mathrm{~mm}$ \\
\hline Diamètre ACross scan (AC) & 800 & $\mathrm{~mm}$ \\
\hline Field AL & 5.9 & $\mathrm{deg}$ \\
\hline Field AC & 3.9 & $\mathrm{deg}$ \\
\hline Total FOV & 23.1 & $\mathrm{deg}$ \\
\hline Focal length & 3500 & $\mathrm{~mm}$ \\
\hline
\end{tabular}

\begin{tabular}{|c|c|c|}
\hline \multicolumn{3}{|l|}{ Focal planes } \\
\hline \multicolumn{3}{|l|}{ CCD detectors } \\
\hline Pixel size $(\mathrm{AL} \times \mathrm{AC})$ & $10 \times 30$ & $\mu \mathrm{m} x \mu \mathrm{m}$ \\
\hline Number of pixels (AL x AC) & $4500 \times 1966$ & \\
\hline Number of detectors AC & 4 & \\
\hline Number of detectors AL & 8 & \\
\hline Number of detectors per telescope & 32 & \\
\hline Total number of detectors & 96 & \\
\hline Operating temperature & $\sim 170$ & $\mathrm{~K}$ \\
\hline Pixel size on the sky (AL x AC) & $0.59 \times 1.77$ & $\operatorname{arcsec}$ \\
\hline Pixel binning (AL $x \mathrm{AC})$ & $21 \times 7$ & \\
\hline Macropixel on the sky (AL x AC) & $12.4 \times 12.4$ & $\operatorname{arcsec}$ \\
\hline Macropixel full well capacity & $2.9 \mathrm{E}+07$ & $\mathrm{e}-$ \\
\hline
\end{tabular}

\begin{tabular}{|l|c|c|}
\hline \multicolumn{3}{|c|}{ Search mode ( Galactic disk scan) } \\
\hline Rotation period & 20 & $\mathrm{mn}$ \\
\hline Scan rate & 0.30 & $\mathrm{deg} / \mathrm{s}$ \\
\hline Explored field of view & 1414 & $\mathrm{deg}$ \\
\hline Detector operation & TDImode & \\
\hline TDI step & 0.55 & $\mathrm{~ms}$ \\
\hline Integration time per detector & 2.46 & $\mathrm{~s}$ \\
\hline Integration time per telescope & 19.6 & $\mathrm{~s}$ \\
\hline Collected signal per hour, V $=11$ & $1.5 \mathrm{E}+08$ & $\mathrm{e}-/ \mathrm{h}$ \\
\hline 1/SNR, per hour, V $=11$ & $8.1 \mathrm{E}-05$ & \\
\hline Number of observed stars, V $=11$ & 144000 & $\mathrm{stars}$ \\
\hline Number of observed stars, V $=14$ & 2500000 & $\mathrm{stars}$ \\
\hline Data rate & 130 & $\mathrm{kbits} / \mathrm{s}$ \\
\hline
\end{tabular}




\begin{tabular}{|l|c|c|}
\hline \multicolumn{2}{|c|}{ Fine observing mode } \\
\hline Field of view per telescope & 23 & deg \\
\hline Total FOV & 69 & deg \\
\hline Detector operation & Frame transfer mode & \\
\hline Observation duration (typical) & 30 & days \\
\hline Integration time (typical, V =11) & 30 & $\mathrm{~s}$ \\
\hline Number of observed stars, V $=11$ & 85000 & stars \\
\hline Number of observed stars, V $=14$ & 1300000 & stars \\
\hline Fraction of galactic disk covered & $1 / 3$ & \\
\hline Data rate & 60 & $\mathrm{kbits} / \mathrm{s}$ \\
\hline
\end{tabular}

\subsubsection{Payload definition}

The three telescopes are mounted on a common torus structure and use separate focal planes. The mirrors and the torus are made of silicon carbide, providing low mass and high stability. The telescope mirrors and the instrument structure are within current manufacturing capabilities. Figure 9 shows the proposed payload.

The telescope optics is constituted of three aspheric mirrors (Fig. 10). The pupil $\left(0.9 \times 0.8 \mathrm{~m}^{2}\right)$ has been located on the primary mirror and the intermirror distance was set about $2.2 \mathrm{~m}$ for accommodation purpose. The largest mirror is the tertiary M3 $\left(\approx 1.6 \times 1.2 \mathrm{~m}^{2}\right)$.

Colour information can be obtained by locating a disperser close the focal plane.

The focal plane assemblies can be based on a strict re-use of the existing Gaia CCD. In fine observing mode, the transfer time is small in comparison to the integration time $(\leq 10 \%)$ and the observation can be achieved without implementing a shutter, as already demonstrated in Eddington studies. The

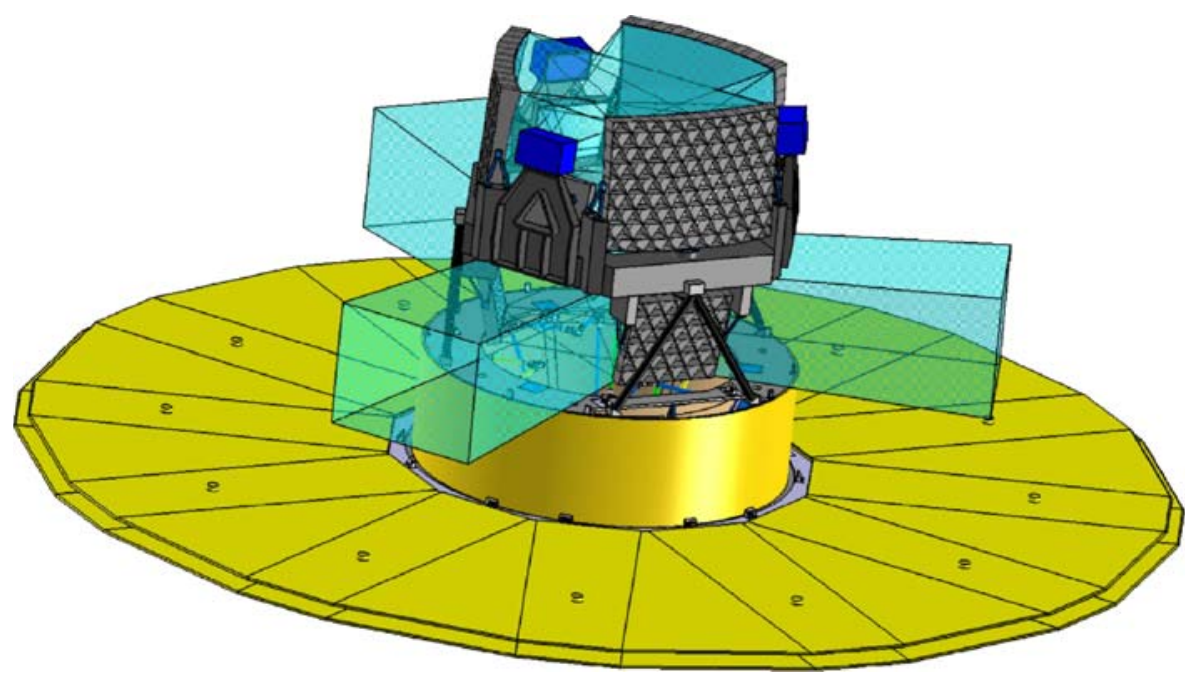

Fig. 9 Spacecraft view for the spinning concept 


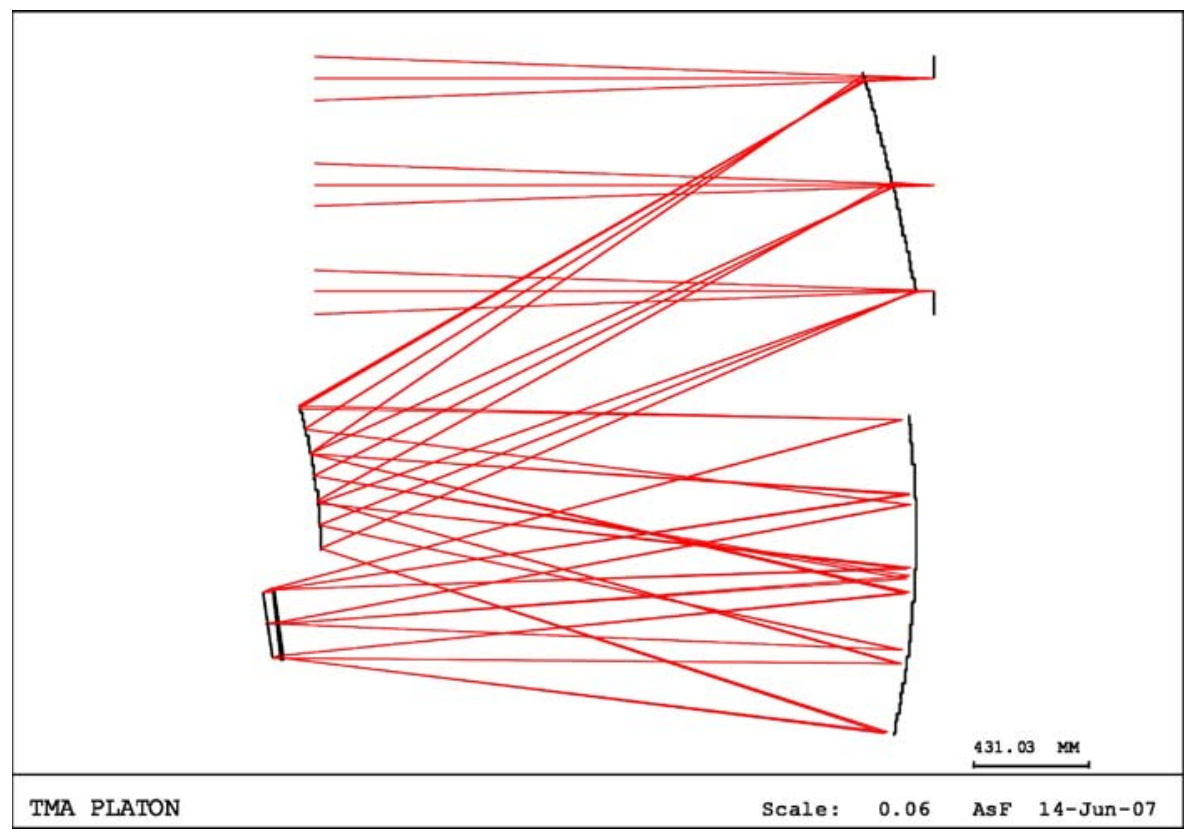

Fig. 10 Optical design

Gaia CCD TDI gates can be used for reducing the integration time and managing the star magnitude dynamical range from $\mathrm{m}_{V}=4$ to 14 , without detector saturation. TDI gates are also useful in fine mode for star position measurements at a high rate $(\approx 1 \mathrm{~Hz})$ for attitude control purpose.

\subsubsection{Expected performance}

The overall performances of the "spinning" concept can be estimated in a straightforward way and are given in Table 3 , in terms of the expected number of stars observed at different noise levels both in search and fine modes.

As for the "staring" concept, we can achieve a very high photometric and astrometric precision for the observation of very bright stars. This approach will be particularly attractive in "search" mode when the whole galactic plane

Table 3 Number of stars observable with various levels of photometric noise

\begin{tabular}{lrrr}
\hline Noise & \# Stars & Mag Dwarfs & \\
\hline $\mathrm{ppm} / \mathrm{h} \quad \mathrm{ppm} / 30 \mathrm{~d}$ & & & \\
\hline \multicolumn{4}{l}{ Search mode, duration } \\
50 & 5525 days & & \\
51 & 144,000 & 28,900 & 10 \\
81 & 240,000 & 125,000 & 11 \\
130 & 85,000 & 11.5 \\
Fine mode, duration 30 days & & \\
20 & 0.7 & 44,000 & 11 \\
\hline
\end{tabular}


Table 4 Bright stars observable with PLATO in the spinning concept

\begin{tabular}{lclll}
\hline $\mathrm{m}_{V}$ & \# Stars & Noise & & $\begin{array}{l}\text { Astrom. noise/ } \\
30 \mathrm{~d}(\mu \text { as })\end{array}$ \\
\cline { 3 - 4 } & & $\mathrm{ppm} / \mathrm{hr}$ & $\mathrm{ppm} / 30 \mathrm{~d}$ & \\
\hline 6 & 290 & 9 & 0.4 & 8 \\
7 & 930 & 15 & 0.7 & 14 \\
8 & 2740 & 24 & 1.1 & 21 \\
\hline
\end{tabular}

will be surveyed, and the use of programmable gates in the CCD TDI process will allow us to very bright stars without saturating the detectors. Table 4 summarizes the expected performance.

\subsection{Advantages of the two concepts}

Sections 5.1 and 5.2 show that two different instrumental concepts comply with the scientific requirements of PLATO. Both are technologically mature, and can be developed at essentially no risk. In this section, we emphasize the advantages of each one of these concepts.

The staring concept is based on a design with a large number of identical telescopes. The payload therefore has a very high level of redundancy, and is very robust against failures or defects of one or even several units. The basic observation strategy, which consists of monitoring continuously the same field for several years, with a duty cycle close to $100 \%$ and an extreme stability, allows to reach a very low level of noise. More than 100,000 stars can be observed at a noise level below $2.5 \times 10^{-5}$ per hour, while about 400,000 stars are monitored at a level $\leq 8 \times 10^{-5}$ per hour. This very low noise on such a high number of targets will allow the detection of exoplanets with sizes significantly smaller than the Earth's, and also the detection of terrestrial planets around stars hotter than the Sun, of spectral type early-F or late-A, thus extending very significantly our statistical knowledge of exoplanet distribution. This concept also enables asteroseismic analysis of all stars monitored by the mission, simultaneously with the transit search.

The spinning concept relies directly on Gaia heritage, is highly recurrent with this previous mission, and offers a large degree of redundancy thanks to the use of 96 CCDs. Its main advantage is to allow the detection of exoplanets in all regions of the galaxy, thanks to the scanning strategy, and therefore to study if and how exoplanet distribution depends on the location in the galaxy. At a given magnitude, it will also survey a somewhat larger number of stars than the staring concept, although at lower precision in search mode (i.e. in the spinning phase). In particular the spinning concept will allow planet transit search on a large number of very bright and nearby stars.

The existence of two different concepts, which are both a priori within the financial limit of a class-M mission, gives a high confidence on the feasibility of the PLATO mission. The choice of concept will be made early in the 
Table 5 Comparison of performance of CoRoT, Kepler, and the two instrumental concepts envisaged for PLATO

\begin{tabular}{llrlrrrr}
\hline Mission & $\begin{array}{l}\text { Coll. area } \\
\left(\mathrm{m}^{2}\right)\end{array}$ & $\begin{array}{l}\text { f.o.v. } \\
\left(\mathrm{deg}^{2}\right)\end{array}$ & $\begin{array}{l}\text { Monitoring } \\
\text { (days) }\end{array}$ & $\begin{array}{l}\text { \# Stars } \\
\text { exo }\end{array}$ & $\begin{array}{l}\text { Mag range } \\
\text { exo }\end{array}$ & $\begin{array}{l}\text { \# Stars } \\
\text { seismo }\end{array}$ & $\begin{array}{l}\text { Mag range } \\
\text { seismo }\end{array}$ \\
\hline CoRoT & 0.057 & 4 & 150 & 60,000 & $11.5-15.5$ & 100 & $5.5-9.5$ \\
Kepler & 0.7 & 111 & 1460 & 100,000 & $9-14$ & 500 & $9-11$ \\
PLATO 1 & 0.75 & 400 & 1825 & 100,000 & $4-12$ & 100,000 & $4-12$ \\
'staring' & & & & 400,000 & $12-14$ & & \\
PLATO 2 & 0.72 & 1240 & 1825 & 144,000 & $4-11$ & 144,000 & $4-11$ \\
'spinning' & & & 20 s every 7 min & $2,500,000$ & $11-14$ & & \\
\hline
\end{tabular}

assessment phase, after an initial evaluation of both solutions, on both scientific and technological points of view.

\section{Comparison with existing \& planned missions}

Table 5 compares the characteristics of CoRoT and Kepler with those planned for PLATO.

These previous space missions will leave a strong need for a further mission aiming at establishing a complete and unbiased statistical knowledge of exoplanetary systems. PLATO, by largely extending the results of CoRoT and Kepler in the area of exoplanet search and characterization, and in that of stellar structure and evolution, represents a natural step in our investigation of stellar and planetary system evolution. Filling and extending the important place in the European strategy that was left vacant by the cancellation of Eddington, PLATO will complete our knowledge of the statistics of extrasolar systems and stellar evolution. Hence, flying a mission like PLATO after CoRoT, Kepler and Gaia is a requirement for our understanding of stellar and planetary formation and evolution.

Acknowledgements We warmly thank the staffs of the Thales-Alenia-Space (Cannes, France) and Astrium-Satellites (Toulouse, France) companies, and especially Thierry Viard and Frédéric Safa, for their efficient help in the technical study of the two instrumental concepts presented here.

\section{References}

1. Aerts, C., Thoul, A., Daszynska, J., Scuflaire, R., Waelkens, C., Dupret, M.A., Niemczura, E., Noels, A.: Asteroseismology of HD 129929: core overshooting and nonrigid rotation. Science 300, 1926 (2003)

2. Fridlund, M., Baglin, A., Lochard, J., Conroy, L. (eds.): The CoRoT Mission, Pre-launch Status. ESA Publication Division, ESA Spec. Publ. 1306 (2006)

3. Borucki, W.J., et al.: The Kepler mission: finding the sizes, orbits and frequencies of earth-size and larger extrasolar planets. ASP CS-294, p. 427 (2003)

4. Brown, T.M.: Expected detection and false alarm rates for transiting Jovian planets. ApJ 593, L125 (2003)

5. Carrier, F., Eggenberger, P., Bouchy, F.: New seismological results on the G0 IV $\eta$ Bootis. A\&A 434, 1085 (2005) 
6. Charbonneau, D., Brown, T., Noyes, R., Gilliland, R.: Detection of an extrasolar planet atmosphere. ApJ 568, 377 (2001)

7. Christensen-Dalsgaard, J., Däppen, W., Dziembowski, W.A., Guzik, J.A.: An introduction to helioseismology. In: Ibanoglu, C. (ed.) Variable Stars as Essential Astrophysical Tools, p. 59. Kluwer, Deventer (2000)

8. Clementini, G., Gratton, R.: The oldest stars and the age of the Universe. Eur. Rev. 10(2), 237-248 (2002)

9. Deming, D., Harrington, J., Seager, S., Richardson, L.: Strong infrared emission from the extrasolar planet HD 189733b. ApJ 644, 560 (2006)

10. Garrido, R.: Photometric modal discrimination in $\delta$ Scuti and $\gamma$ Doradus stars. In: Breger, M., Montgomery, M.H. (eds.) $\delta$ Scuti and Related Stars. ASP CS-210, p. 67 (2000)

11. Grillmair, C., Charbonneau, D., Burrows, A., Armus, L., Stauffer, J., Meadows, V., Van Cleve, J., Levine, D.: A spitzer spectrum of the exoplanet HD 189733b. ApJ 658, L115I (2007)

12. Israelian, G., Santos, N.C., Mayor, M., Rebolo, R.: Evidence for planet engulfment by the star HD82943. Nature 411, 163 (2001)

13. Knutson, H., et al.: A map of the day-night contrast of the extrasolar planet HD 189733b. Nature 447, 183 (2007)

14. Krauss, L.M., Chaboyer, B.: Age estimates of globular clusters in the milky way: constraints on cosmology. Science 299, 65 (2003)

15. Mermilliod, J.C., Maeder, A.: Evolution of massive stars - comparison of cluster sequences and models with mass loss. A\&A 158, 45 (1986)

16. Pamyatnykh, A.A., Handler, G., Dziembowski, W.A.: Asteroseismology of the $\beta$ Cephei star $v$ Eridani: interpretation and applications of the oscillation spectrum. MNRAS 350, 1022 (2004)

17. Perryman, M.A.C., Brown, A.G.A., Lebreton, Y., et al.: The Hyades: distance, structure, dynamics, and age. A\&A 331, 81 (1998)

18. Richardson, L., Deming, D., Horning, K., Seager, S., Harrington, J.: A spectrum of an extrasolar planet. Nature 445, 892 (2007)

19. Robin, A.C., Reylé, C., Derrière, S., Picaud, S.: A synthetic view on structure and evolution of the Milky Way. A\&A 409, 523 (2003)

20. Roxburgh, I.W.: Eddington and the internal constitution of the stars in Stellar Structure and Habitable Planet Finding, 2nd Eddington Workshop, ESA-SP, vol. 538, p. 23 (2004)

21. Schneider, J.: Extrasolar planets transits: detection and follow-up. In: Antofagasta, Bergeron, J., Renzini, A. (eds.) Proc. of the VLT Opening Symposium, p. 499. Springer-Verlag, Berlin Heidelberg New York (2000)

22. Udry, S., et al.: The HARPS search for southern extra-solar planets. XI. Super-Earths in a 3-planet system. A\&A 469, L43 (2007)

23. van den Bergh, S.: Ages of the oldest clusters and the age of the universe. Science 270, 1942 (1995)

24. Vidal-Madjar, A., et al.: Detection of oxygen and carbon in the hydrodynamically escaping atmosphere of the extrasolar planet HD 209458b. ApJ 604, L69 (2004) 